Research Paper

\title{
Gene Silencing of Human Neuronal Cells for Drug Addiction Therapy using Anisotropic Nanocrystals
}

\section{Wing-Cheung Law ${ }^{1 *}$, Supriya D. Mahajan ${ }^{2 *}$, Atcha Kopwitthaya ${ }^{1}$, Jessica L. Reynolds ${ }^{2}$, Maixian Liu ${ }^{1}$, Xin

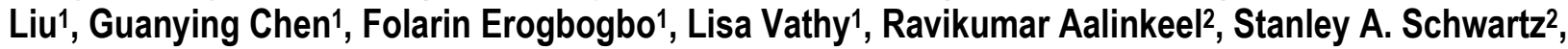 Ken-Tye Yong ${ }^{1,3 凶}$ and Paras N. Prasad ${ }^{1 凶}$}

1. Institute for Lasers, Photonics and Biophotonics, University at Buffalo, State University of New York, Buffalo, NY 14260-4200, USA.

2. Department of Medicine, Division of Allergy, Immunology, and Rheumatology, The State University of New York, Buffalo General Hospital, Buffalo, NY 14203, USA.

3. School of Electrical and Electronic Engineering, Nanyang Technological University, Singapore 639798, Singapore.

* Authors contributed equally.

$\triangle$ Corresponding author: Paras N. Prasad, PhD, 428 Natural Science Complex, University at Buffalo, Buffalo, NY 14260-3000, USA Tel:1-716-645-4147, FAX: 1-716-645-6945, email: pnprasad@buffalo.edu Ken-Tye Yong, PhD, School of Electrical and Electronic Engineering, Nanyang Technological University, Singapore 639798, Singapore Tel: 65-6790-5444, email: ktyong@ntu.edu.sg.

(C) Ivyspring International Publisher. This is an open-access article distributed under the terms of the Creative Commons License (http://creativecommons.org/ licenses/by-nc-nd/3.0/). Reproduction is permitted for personal, noncommercial use, provided that the article is in whole, unmodified, and properly cited.

Received: 2011.08.29; Accepted: 2011.09.20; Published: 2012.07.27

\begin{abstract}
Theranostic platform integrating diagnostic imaging and therapeutic function into a single system has become a new direction of nanoparticle research. In the process of treatment, therapeutic efficacy is monitored. The use of theranostic nanoparticle can add an additional "layer" to keep track on the therapeutic agent such as the pharmacokinetics and biodistribution. In this report, we have developed quantum rod (QR) based formulations for the delivery of small interfering RNAs (siRNAs) to human neuronal cells. PEGlyated QRs with different surface functional groups (amine and maleimide) were designed for selectively down-regulating the dopaminergic signaling pathway which is associated with the drug abuse behavior. We have demonstrated that the DARPP-32 siRNAs were successfully delivered to dopaminergic neuronal (DAN) cells which led to drastic knockdown of specific gene expression by both the electrostatic and covalent bond conjugation regimes. The PEGlyated surface offered high biocompatibilities and negligible cytotoxicities to the QR formulations that may facilitate the in vivo applications of these nanoparticles.
\end{abstract}

Key words: Quantum Rod, Gene Delivery, Addiction Gene Therapy, Phospholipid, PEG, siRNA.

\section{Introduction}

The application of nanotechnology in medicine, or so called nanomedicine, promises quantum leaps in advanced diagnosis and therapy of various disease and/or pathophysiological conditions. Nanoparticle-based disease diagnosis, imaging, therapeutic drug delivery, gene material delivery, immunoassay and biosensor have received remarkable development over the past decade [1-15]. Recently, gene therapy has drawn a lot of attention because of the revolutionary therapeutic strategy and the highly selectivity to a particular disease [16-20]. The discovery of RNA interference (RNAi), which involves an inhibition of specific gene expression in targeted organs or cells using small interfering RNA (siRNA), imparted a new 
approach to overcome the difficulties in conventional methods [21-23]. The use of siRNA as a powerful tool for therapy is attractive and promising. However, owing to the fragile and negatively charged nature of siRNA, it cannot penetrate the cell membrane easily and efficiently. Nanoparticles can form stable complexes with siRNA molecules (nanoplexes), which can tackle various bottlenecks associated with the delivery of siRNA in free form [24-29]. For a successful delivery of siRNA to the nucleus, one should (i) neutralize the negative charge of siRNA, (ii) internalize the siRNAs through cell membrane and (iii) protect the siRNAs from degradation in cytoplasm.

Many non-viral delivery vectors such as Lipofectamine, siPORT and polyethylenimine (PEI) polymer have been developed to satisfy the demand [30-33]. However, the intrinsic cytotoxicity and the high reactivity in physiological environment of these materials have limited their application for further studies (e.g. in vivo) [34, 35]. Indeed, many concerns, such as clearance by the reticuloendothelial system, degradation in the endosomal compartment and interaction with serum proteins, should be addressed before the systemic administration. To tackle these challenges, several strategies have been applied to PEI by masking PEI surface with an additional biocompatible layer, for example, polyethylene glycol (PEG) and poly(lactic-co-glycolic acid) (PLGA) [36]. Typically, electrostatic force has been widely utilized for siRNA conjugation due to the high transfection efficiency and simple preparation protocol. Previously, our group demonstrated that electrostatic conjugation can be an efficient approach for delivering adenosine 3',5' -monophosphate-regulated phosphoprotein (DARPP-32) siRNA- gold nanorod nanoplexes to human neuronal cells [37]. In addition, several research groups demonstrated a covalent bonding approach for the immobilization of siRNA on the nanoparticle surface. Furthermore, quantum dots and siRNAs that were conjugated with a longer linker length would facilitate the formation of RNA-Induced Silencing Complex (RISC) and result in better silencing performances [38]. It was found that spacers should be introduced between nanoparticle and siRNA in order to overcome the steric hindrance and improve the availability of siRNA [39].

A nanotechnology approach that uses targeted siRNA-nanoparticle complexes or nanoplexes has the potential to be developed into a powerful tool to therapeutically modulate various pathological/behavioral conditions in the human brain at the genetic level. This can have the clinical application of siRNA-based therapeutics against not only drug addiction, but also against several other chronic illness- es. In the current report, we present two types of anisotropic nanocrystal i.e. quantum $\operatorname{rod}(\mathrm{QR})$-siRNA nanoplex designs, which are based on phospholipid micellar system, for gene silencing-mediated drug addiction therapy. Since DARPP-32 plays a significant role in substance abuse behavior which involves an activation of the dopaminergic signaling pathway in the brain, the addiction behavior can be controlled by modulating the gene expression of DARPP-32. Quantum rod has become an attractive tool for labeling or carrying biomolecules as it has unique properties over the organic dye, for example, tunable emission wavelength, single excitation source for wide range of emission, less tendency to photobleaching and longer lifetime. Although the uncovered toxicity issue and the heavy-metal containing nature might make CdSe QRs difficult to be transferred to clinical setting, many studies have shown that QR was readily served as an excellent nanoprobe for "proof-of-concept" purpose [40-43]. The phospholipids used in our study (DSPE-PEG-Amine and DSPE-PEG-Maleimide) could be divided into three moieties: (i) DSPE, the hydrophobic part to assemble the QR into aggregates, (ii) PEG, the spacer to overcome the steric barrier and (iii) amine or maleimide, the functional groups for siRNA conjugations by electrostatic force or covalent bond. Phospholipids were chosen in this study because excellent in vivo biocompatibility, drug load capacity and tumor targeting capability of QD-phospholipid complex have been demonstrated by our group. Thus, the designs presented here can be quickly adopted and can offer us an additional "layer" that enables targeted therapy to specific disease besides the use of drug.

\section{Materials and Methods}

\section{Materials}

Cadmium oxide, selenium powder, sulfur, trioctylphosphine oxide (TOPO), trioctylphosphine (TOP), oleic acid, zinc acetate and HPLC water were purchased from Sigma-Aldrich. Tetradecyl phosphonic acid (TDPA) was obtained from Alfa Aesar. DSPE-PEG-Maleimide MW 3400 and DSPE-PEG-NH2 MW 3400 were purchased from Laysan Bio Inc. 1,2-distearoyl-sn-glycero-3phosphoethanolamine-N-[methoxy(polyethylene glycol)-2000] (ammonium salt) (PEG2000 PE) was purchased from Avanti Polar Lipids, Inc. Poly(diallydimethylammonium chloride) (PDDAC) was obtained from Polysciences, Inc. Negatively charged siRNA (sense: :ACA CAC CAC CUU CGC UGA AAG CUG U; antisense: ACA GCU UUC AGCGAAGGUGGUGUGU) and thiolated siRNA 
(sense:/5BHQ_1/ACA CAC CACCUU CGC UGA AAG CUG U/iSp18//3ThioMC3-D/ and antisense: ACA GCU UUC AGCGAAGGUGGUGUGU/ 36-FAM/) for DARPP-32 were purchased from IDT technologies. All chemicals were used as received.

\section{Synthesis of CdSe/CdS/ZnS QRs}

The CdSe quantum rods were prepared by dissolving $1.6 \mathrm{mmol}$ of cadmium oxide, $3 \mathrm{mmol}$ of TDPA, and $3 \mathrm{~g}$ of TOPO into a $100 \mathrm{ml}$ three-necked flask. The reaction mixture was slowly heated under an argon atmosphere to $150-160^{\circ} \mathrm{C}$ for 2 hours. The temperature was then set to $320^{\circ} \mathrm{C}$ for 5 minutes followed by rapid injection of $0.8 \mathrm{ml}$ of $1 \mathrm{M}$ TOP-Se. The reaction was stopped after 3-5 minutes by removing the heating mantle and addition of toluene. The QRs were separated from the surfactants solution by addition of ethanol and centrifugation.

The synthesis of CdS/ZnS graded shell on CdSe QR was obtained by dissolving $2 \mathrm{mmol}$ of cadmium oxide, $4 \mathrm{mmol}$ of zinc acetate, $5.5 \mathrm{~g}$ of TOPO and 10 $\mathrm{mL}$ of oleic acid into a $100 \mathrm{ml}$ three-necked flask. The reaction mixture was heated to $120^{\circ} \mathrm{C}$ for $30 \mathrm{~min}$ under an argon flow, and then the CdSe NR solution was injected slowly under stirring into the hot reaction mixture. The reaction mixture was held at $120{ }^{\circ} \mathrm{C}$, with a needle outlet that allowed the solvent to evaporate. After $15 \mathrm{~min}$ of heating, the needle was removed, and the reaction temperature was raised to $210{ }^{\circ} \mathrm{C}$. When the desires temperature was reached, 2 $\mathrm{mL}$ of TOP-S was added dropwise into the reaction mixture. The reaction mixture was then held at $210{ }^{\circ} \mathrm{C}$ for $10-15 \mathrm{~min}$. The QRs were purified by the addition of ethanol and centrifugation.

\section{Characterizations of $Q R$}

The absorption spectra of QRs were collected using a Shimadzu model 3101PC UV-vis-near infrared (NIR) scanning spectrophotometer over a wavelength range from 400 to $800 \mathrm{~nm}$. The $\mathrm{QR}$ emission spectra were collected using a Fluorolog-3 spectrofluorometer (Jobin Yvon; fluorescence spectra). Quantum yields (QYs) of the QR dispersions were determined by comparing the integrated emission from the QRs to rhodamine 6G dye solutions (QY: $90 \%$ ) of matched absorbance. Samples were diluted to ensure that there was no "self-absorption". For particle size determination, high-resolution transmission electron microscopy (HRTEM) images were obtained using a JEOL model JEM 2010 microscope at an acceleration voltage of $200 \mathrm{kV}$. The specimens were prepared by drop-coating the sample dispersion onto an amorphous carbon-coated 300 mesh copper grid, which was placed on filter paper to absorb excess solvent. The size distribution and surface potential of QRs were determined by dynamic light scattering (DLS) measurement with a Brookhaven Instruments 90Plus particle size analyzer, with a scattering angle of 90. A phase analysis light scattering module was used for the zeta-potential measurement.

\section{Preparation of $\mathrm{NH}_{2}$ and Mal terminated water dispersible QRs}

Water dispersible QRs were prepared using phospholipid encapsulation. Briefly, QR suspension $(\sim 4 \mathrm{mg} / \mathrm{ml}$ in chloroform), mPEG2000 solution $\left(\sim 20 \mathrm{mg} / \mathrm{ml}\right.$ in chloroform), DSPE-PEG-NH${ }_{2}$ solution $(\sim 10 \mathrm{mg} / \mathrm{ml}$ in chloroform) and DEPE-PEG-Mal solution $(\sim 10 \mathrm{mg} / \mathrm{ml}$ in chloroform) were prepared as the stock solutions. Mixture of QR $(100 \mu \mathrm{l})$, mPEG2000 $(100 \mu \mathrm{l})$ and DSPE-PEG-NH $\mathrm{NH}_{2}(100 \mu \mathrm{l})$ and mixture of

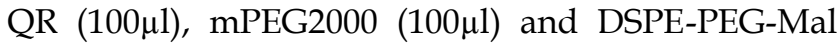
$(100 \mu \mathrm{l})$ were loaded in $10 \mathrm{ml}$ round bottomed flasks separately. Chloroform was then removed slowly using a rotary evaporator. After evaporation, $\sim 3.4 \mathrm{ml}$ of HPLC water was added to the dry film followed by gentle stirring. The final concentrations of water suspensions ( $\mathrm{QR}^{\mathrm{NH} 2}$ and $\left.\mathrm{QR}^{\mathrm{mal}}\right)$ were regarded as $1 \mathrm{mg} / \mathrm{ml}$. Preparation of positively charged water dispersible QRs $\left(\mathrm{QR}^{+}\right)$was obtained by adding 10ul of PDDAC to $10 \mathrm{ml}$ of $\mathrm{QR}^{\mathrm{NH} 2}$ dispersion under vigorous stirring for 4 hours. The samples were purified by dialysis against HPLC water. The concentration can be maintained by removing excess water using vacuum pump.

\section{Conjugation of water dispersible QRs with SiRNA}

A $30 \mu \mathrm{l}$ of $\mathrm{QR}^{+}$stock solution was mixed with $10 \mu \mathrm{l}$ of DARPP32-siRNA $(10 \mu \mathrm{M})$ (weight of $\mathrm{QR} /$ weight of siRNA ratio $=23$ ) with gentle vortex. The mixture was left undisturbed for 30 minutes ( $\mathrm{QR}^{+}$-siRNA nanoplex). For QRmal-thiosiRNA, 30 $\mu \mathrm{l}$ of $\mathrm{QR}$ mal stock solution was mixed with $10 \mu$ l of thiolated DARPP32-siRNA $(10 \mu \mathrm{M})$ (weight of QR/weight of siRNA ratio $=23$ ) with gentle vortex. The mixture was left undisturbed for 30 minutes (QRmal-thiosiRNA nanoplex).

\section{Cell Culture}

Human dopaminergic neuronal (DAN) cells were obtained from Clonexpress. Actively growing populations of cells were tested for tyrosine hydroxylase $(\mathrm{TH})$ expression by immunocytochemistry. DAN cells are supplied with a proprietary growth factor supplement (DNCS) as a 100xstock solution, which is added to DMEM:F12 (50:50) containing 5\% FBS and $10 \mathrm{ng} / \mathrm{ml}$ of bFGF and $5 \mathrm{ng} / \mathrm{ml}$ of GDNF to 
make DAN cell growth medium. These cells differentiate into neurons within a week, when plated on polylysine (PLL)-coated plates at a density of approximately 104 cells $/ \mathrm{cm}^{2}$ in DMEM:F12 (50:50) supplemented with DNC5, 5\% FBS, $10 \mathrm{ng} / \mathrm{ml}$ of bFGF, 10 $\mathrm{ng} / \mathrm{ml}$ of EGF, and $100 \mu \mathrm{M}$ dibuturyl cAMP.

\section{Cell Viability Study}

Cell viability assay measures the reduction of a tetrazolium component 3-(4, 5-dimethylthiazol-2-yl)5-(3-carboxymethoxyphenyl)-2-(4-sulfophenyl)-2H-te trazolium, or MTS into a formazan product by the mitochondria of viable cells. DAN cells in a 96-well plate $(\sim 5,000$ cells/well) were incubated with different concentrations of QR+ and $\mathrm{QR}^{\mathrm{mal}}$ for 48 and 72 hours at $37^{\circ} \mathrm{C}$ under $5 \% \mathrm{CO}_{2}$. The untreated cell was served as the control. The absorbance of formazan (produced by the cleavage of MTS by dehydrogenases in living cells) is directly proportional to the number of live cells. After the incubation, MTS reagent was added to each well. After 1 hour, the absorbance of sample at $490 \mathrm{~nm}$ was recorded using multiwell plate reader (Opsys MR ${ }^{\mathrm{TM}}$, Dynex).

\section{Transfection}

Transgene expression was monitored at 72 hours post-treatment. 24 hours before siRNA transfection, $\sim 20,000$ dopaminergic neuronal cells were seeded onto 6-well plates in OPTI-MEM containing 4\% FBS with no antibiotics to give 30 to $50 \%$ confluence at the time of transfections. The siRNA was reconstituted in DNase-RNase free water to a final concentration of $10 \mu \mathrm{M}$ and mixed with $20 \mu \mathrm{l}$ of QR solutions as described above. The final concentration of siRNA for in vitro transfection of dopaminergic neurons was 100nM. The commercially available siRNA delivery agent, Lipofectamine (Invitrogen) was used as the positive control in our experiments.

\section{RNA Extraction}

Cytoplasmic RNA was extracted by an acid guanidinium-thiocyanate-phenol-chloroform method as described using TRIzol reagent (Invitrogen-Life Technologies). The amount of RNA is quantitated using a Nano-Drop ND-1000 spectrophotometer (Nano-Drop). Real-time quantitative PCR was used to determine the relative abundance of each mRNA species. RNA was reverse transcribed to cDNA using the reverse transcriptase kit from Promega. Relative expression of mRNA species was calculated using the comparative threshold cycle number (CT) method.

\section{Results and Discussion}

The CdSe/CdS/ZnS QRs were synthesized us- ing well-established procedures as described in our previous studies [42]. The as-prepared nanocrystals exhibit narrow emission range (full width at half maximum $\sim 28 \mathrm{~nm}$ ) along with high photoluminescence (PL) quantum yield (QY $\sim 45 \%$ ). The CdS/ZnS graded shell has served as an efficient protective layer to CdSe QR for enhancing QY without introducing lattice strain. Figure 1 shows the UV absorption and emission profiles of QRs. The absorption spectrum illustrates a typical excitonic band of QR. The emission peak is $\sim 633 \mathrm{~nm}$ which is suitable for bioimaging as it can be easily distinguished from the autofluorescence in biological samples. The inset shows a TEM picture of monodispersied QRs, the length and the diameter of QR were estimated as $12.6 \pm 0.9 \mathrm{~nm}$ and $4.8 \pm 0.6 \mathrm{~nm}$ respectively. A photograph of highly luminescent QR solution (in chloroform), which was exposed to a hand-held UV lamp under room light, is also shown in the inset. Two water dispersible formulations with different surface functional groups (amine and maleimide) were prepared using phophoslipids. Recently, many reports have revealed that QRs conferred several important advantages as bioimaging probes over spherical QDs. The unique properties of QRs such as linearly polarized emission, large surface area, high quantum yield and lasing capability made QRs promising nanoprobes for both in vitro/in vivo bioimaging and single molecule detection [40, 44, 45]. Our group has demonstrated the use of CdSe/CdS/ZnS QRs as efficient probes for cancer cell labeling, blood brain barrier trafficking and in vivo tumor targeting [42, 46-48]. Moreover, QR was not limited to be a diagnostic tool. To fully explore the potential of $\mathrm{QR}$ to be a theranostics reagent, herein, we report two designs of QR nanosystems which can be readily served as gene delivery vehicles as well as high luminescence imaging probes.

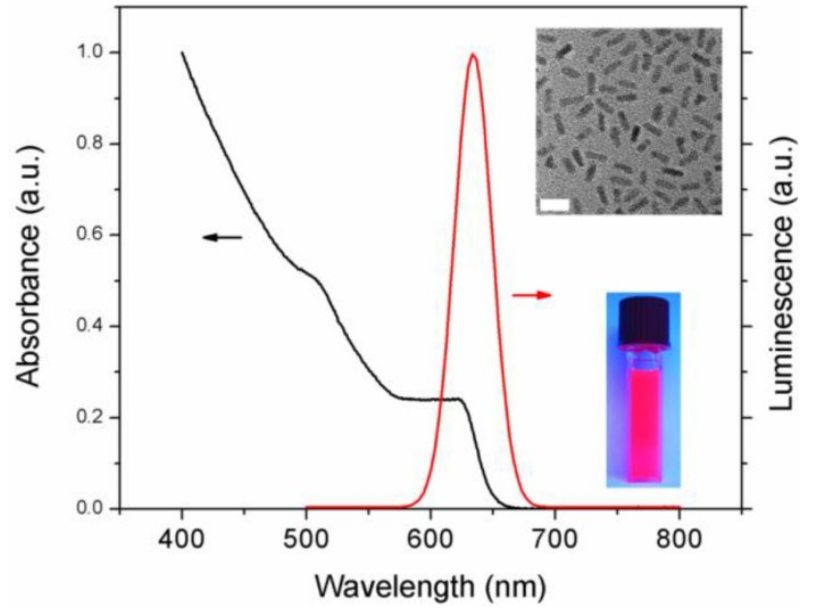

Figure I. Absorption and emission profiles of QR. A TEM picture (scale bar: $20 \mathrm{~nm}$ ) and a photograph of QR are shown in the inset. 
Scheme 1 (Figure S1) describes the concept of gene therapy for drug addiction utilizing QR-siRNA nanoplexes and our approaches. Neurotransmitters such as dopamine activate protein kinase A (PKA) mediated phosphorylation of DARPP-32. Both dopamine and glutamate receptors have been implicated in inducing modulation of DARPP-32, resulting in the activation of the extracellular signal-regulated kinase (ERK) mitogen-activated protein (MAP) kinase cascades. ERK activity has been known to be important in neuronal plasticity and its pharmacologic blockade prevents the transcriptional and behavioral effects of various drugs of abuse. Thus, it is believed that DARPP-32 is the central molecular "trigger" that underlies the neurobiological alterations related to drug abuse. Therefore, we hypothesize that the suppression of DARPP-32 gene expression using delivery of its antagonist short interfering RNA (siRNA) will lead to a radical new approach for the treatment of drug addiction. The suppression of DARPP-32 gene expression in the brain as well as the key downstream effector molecules such as protein phosphatase-1 (PP-1) via the activation of ERK can effectively decrease the activation of the dopaminergic signaling pathway which may lead to significant inhibition of the addiction behavior (Scheme 1a). In order to develop an efficient and traceable nanocarrier for DARPP-32 gene delivery, in Scheme 1b, we proposed two types of nanoformulations which utilize two different functional groups terminated (amine and maleimide) phospholipids for surface passivations. TOPO-coated QR interacted with the hydrophobic component of phospholipid and was encapsulated in the core. A micelle-structure was formed with a PEG-rich outer layer. This layer not only facilitated the water dispersibility, impeded the degradation and absorption in the biological environment, especially for in vivo studies, but also acted as a spacer which could be an essential component for gene delivery. The terminated functional groups of phospholipids rendered the versatilities for siRNA conjugations. It is important to know that the functionalized phospholipids with longer PEG chains have been chosen in our design. Especially for $\mathrm{QR}^{+}$, the longer $\mathrm{NH}_{2}$ terminated arm was served as an anchor for further conjugation of targeting molecules such as antibodies and peptides and reduces the possibility of PDDAC coverage. As shown in Scheme $1 b$, the presence of amine groups with PDDAC rendered the QR positively surface charge which can be used for efficient siRNA/targeting molecule conjugation by the electrostatic force and carbodiimide chemistry. In addition, another design for obtaining stable siRNA-QR complex was based on the robust carbon-sulfur bond formed between the thiol-modified siRNA and the maleimide group terminated QR surface. This design is more favorable for in vivo application since the covalent bond is strong enough to remain intact in the biological environment during circulation.

(a)

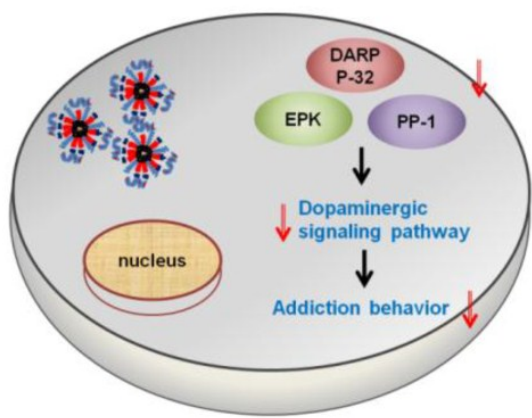

(b)



Figure SI. (Scheme I). (a) Gene therapy illustration. The delivery of QR-siRNA nanoplexes suppress the expression of DARPP-32, EPK, and PP-I protein which belong to dopaminergic signaling pathway. The reduced activation of this pathway results in less addiction behavior. (b) siRNAs were conjugated to $Q R$ by the electrostatic force $\left(\mathrm{QR}^{+}\right)$and the carbon-sulfur bond ( $\mathrm{QR}$ mal).

The use of DSPE-PEGs as the surface coatings in our studies was due to the fact that PEG-modified nanoparticles were proven to be able to provide high stability of nanoparticles and prevent their rapid clearance by the reticuloendothelial system (RES) by imparting a steric barrier to opsonization. In addition, different functional groups (carboxyl, amino, thiol, biotin, maleimide, etc) modified DSPE-PEGs are commercially available and have been utilized in a wide range of bio-applications. In our micellar encapsulation strategies, the hydrophobic (DSPE) part assembled the QR into aggregates which can be eventually dispersed in aqueous media with the aid of PEG hydrophilic group. Since the water solubilities of amino- and maleimide-PEG are different, their self-oriented nanomicelles ( $\mathrm{QR}^{\mathrm{NH} 2}$ and $\mathrm{QR}^{\mathrm{Mal}}$ ) possessed different sizes and charge properties. As revealed in Table 1, the size of QR-nanomicelles could have a large range of variation from $\sim 65$ to $\sim 200 \mathrm{~nm}$. The larger hydrodynamic diameter observed in $\mathrm{QR}{ }^{\mathrm{mal}}$ nanomicelle was due to the poorer solubility of ma- 
leimide functional group. It is also noted that $\mathrm{QR}^{\mathrm{NH}} \mathrm{2}$ possessed negative charge even though the cationic amino groups attributed the positive charges to the nanomicelle. Furthermore, the net negative charge of $\mathrm{QR}^{\mathrm{NH}}$ 2, which originated from the PEG chain, can be reversed by the addition of PDDAC layer.

Table I. Hydrodynamic diameter and zeta potential of different QR nanomicelles.

\begin{tabular}{lll}
\hline Sample & Hydrodynamic diameter $(\mathrm{nm})$ & Zeta potential $(\mathrm{mV})$ \\
\hline $\mathrm{QR}^{\mathrm{NH} 2}$ & 65.5 & -18.8 \\
$\mathrm{QR}^{+}$ & 66.8 & +25.4 \\
$\mathrm{QR}^{\mathrm{mal}}$ & 195.6 & -37.7 \\
\hline
\end{tabular}

The colloidal stability of nanoparticle formulation is known to be an important aspect in both in vitro and in vivo applications. Dynamic light scattering was used as an accurate approach to monitor the hydrodynamic diameter of nanoparticle formulation in different buffer (water and DMEM). As shown in Figure 2 , the hydrodynamic diameter of $\mathrm{QR}^{+}$and $\mathrm{QR}^{\mathrm{mal}}$ nanoparticle were $\sim 65 \mathrm{~nm}$ and $\sim 200 \mathrm{~nm}$, respectively. It should be also noted that the variations of hydrodynamic diameter were less than $20 \%$ over 2 days, suggesting that their colloidal stability is not affected in the biological buffer.

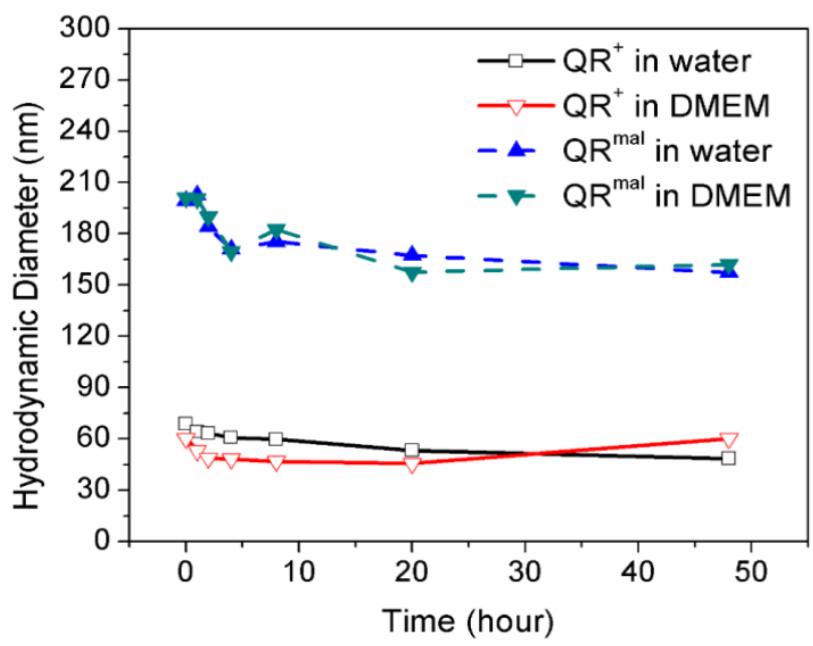

Figure 2. Stability of $Q R$ nanomicelles in phosphate buffered saline (PBS) and Dulbecco's modification of Eagle's medium (DMEM) over time

Figure 3 shows the confocal images of neuronal cells (DAN) treated with (a) FAM labeled siRNA (siRNA ${ }^{\text {FAM }}$ ), (b) Lipofectamine-siRNA ${ }^{\text {FAM }}$ (Lipo-siRNA ${ }^{\text {FAM }}$ ) nanoplex, (c) $\mathrm{QR}^{+}$nanomicelle, (d) siRNAFAM conjugated $\mathrm{QR}^{+}$nanomicelle
(QR-siRNAFAM) and (e) thiolated siRNAFAM conjugated with $\mathrm{QR}^{\text {mal }}$ (QR Q $\left.^{\text {mal-thiosiRNA }}{ }^{\mathrm{FAM}}\right)$ for 20 hours. Figure 3a shows minimal uptake of unprotected siRNA. It can be due to the fact that the uptake of negatively charged siRNA was impeded by the cell membrane and the fast degradation of unprotected siRNA in the endosomal compartment. Figure $3 b$ shows the uptake of Lipofectamine anchored siRNA ${ }^{\text {FAM }}$ (green channel). Furthermore, strong fluorescence (red channel), which associated with the excited luminescence from the QR, is observed in Figure 3c. The significant internalization of QR should allow us to traffic siRNA into the cells and prevent them from the fast degradation. This concept was demonstrated in Figure 3d and 3e. As shown in the first two columns of Figure $3 \mathrm{~d}$ and $3 \mathrm{e}$, fluorescence signals from siRNA ${ }^{\mathrm{FAM}}$ and $\mathrm{QR}$ were detected simultaneously, indicating the proper trafficking of siRNA within the cells using different QR nanomicelles. The overlaid images in the fourth column reveal that most of the QR-siRNAFAM nanoplexes were being uptaken into the cytoplasm through the process endocytosis. The fluorescence from both $\mathrm{QR}$ and siRNA ${ }^{\mathrm{FAM}}$ appeared to accumulate around the perinuclear envelop, indicating that QR nanomicelle can facilitate intracellular uptake of siRNA. It is worth mentioning that the thiolated siRNA was specially designed in which the sense strand of RNA was modified with a thiol group and a fluorescence quencher (5BHQ_1) while the antisense was labeled with FAM (see Materials). When they were packed together, the fluorescence of FAM was quenched. By monitoring the FAM fluorescence from the antisense strand, one can track the intracellular unwinding of antisense strand from the siRNA in the process of forming the RNA-induced silencing complex.

To elucidate the effect of QR-siRNA nanoplex on gene silencing for the purpose of drug addiction therapy, functional siRNAs specific for DARPP-32 (siRNA ${ }^{\mathrm{D}-32}$ ) silencing were conjugated to $\mathrm{QR}$ nanomicelles through the electrostatic force and the covalent bond as mentioned above. The weight ratio of nanomicelle and siRNA was maintained at 23 (see Materials and Methods section). DAN cells were incubated with $\mathrm{QR}^{+}$-siRNA ${ }^{\mathrm{D}-32}$ and $\mathrm{QR}^{\text {mal }}$-thiosiRNA ${ }^{\mathrm{D}-32}$ nanoplexes for 48 hours and the efficiency of gene silencing was determined by measuring the percentage inhibition of the expression of DARPP-32 using quantitative real-time (Q)-PCR. In our previous report, we showed that the knockdown of DARPP-32 gene led to the down-regulation of on effector molecules such as PP-1 and ERK in the opiate signaling pathway and thus inhibited the development of drug addiction. The efficiency of DARPP-32 suppression of 
$\mathrm{QR}^{+}$-siRNA ${ }^{\mathrm{D}-32}$ and $\mathrm{QR}^{\text {mal }}$-thiosiRNA ${ }^{\mathrm{D}-32}$ were compared with commercially available reagent Lipofectamine. Our results (Figure 4) show that the cells treated with free siRNA ${ }^{\mathrm{D}-32}$ and QR only show minimal suppressions while the suppressions obtained from the cells treated with $\mathrm{QR}^{+}$-siRNA ${ }^{\mathrm{D}-32}(69 \%$ knockdown) and QRmal-thiosiRNA ${ }^{\mathrm{D}-32}$ (61\% knockdown), indicating that the gene silencing efficiencies of QR-based micelles were comparable to the Lipofectamine (55\% knockdown).

When a nanoparticle system is designated for therapeutic applications, one should be extra vigilant about the intrinsic cytotoxicity of nanoparticle. Many reports suggested that the use of Lipofectamine for gene silencing is limited to in vitro due to the high toxicity $[34,35,49]$. In our case, MTS assay was used to evaluate the cytotoxicity of $\mathrm{QR}^{+}$and $\mathrm{QR}^{\text {mal }}$ nanomicelles. Figure 5 shows the results of a cell viability assay following treatment of DAN cells with QR nanomicelles for 48 and 72 hours. It was found that all the nanomicelles are non-toxic even at the concentration as high as $500 \mu \mathrm{g} / \mathrm{ml}$, indicating that phospholipid-PEG exerted an excellent protective function to the hydrophobic core so that the QRs remained intact in the biological buffer. Thus, the low cytotoxicity and the high gene transfection rate of these QR-siRNA nanoplexes offer a combination of diagnostic and therapeutic modalities in biomedical imaging and gene therapy.



Figure 3. Confocal images of neuronal cells (DAN) treated with (a) FAM labeled siRNA (siRNAFAM), (b) Lipofectamine-siRNAFAM (Lipo-siRNAFAM) nanoplex, (c) QR ${ }^{+}$nanomicelle, (d) siRNAFAM conjugated $\mathrm{QR}^{+}$nanomicelle $\left(\mathrm{QR}^{+}\right.$-siRNAFAM) and (e) thiolated siRNAFAM conjugated with $Q R^{\text {mal }}$ (QRmal-thiosiRNAFAM). The pseudo-colors assigned for $Q R$ and siRNAFAM are red and green respectively (Yellow: red overlaid with green). 


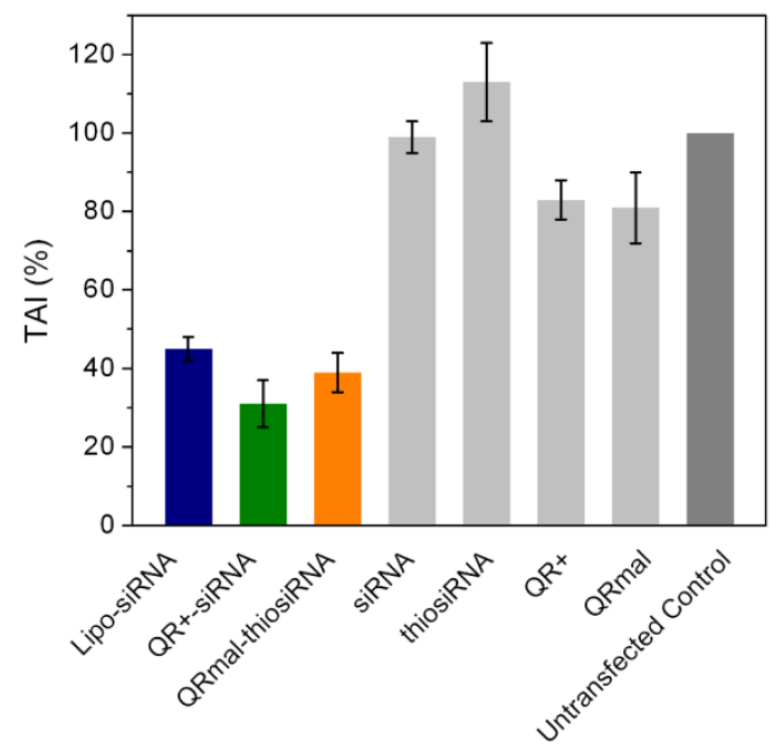

Figure 4. Transfection effect of QR-siRNA nanoplex in DAN cells for DARPP-32 gene silencing (TAl:Transcript accumulation index)

(a)

(b)
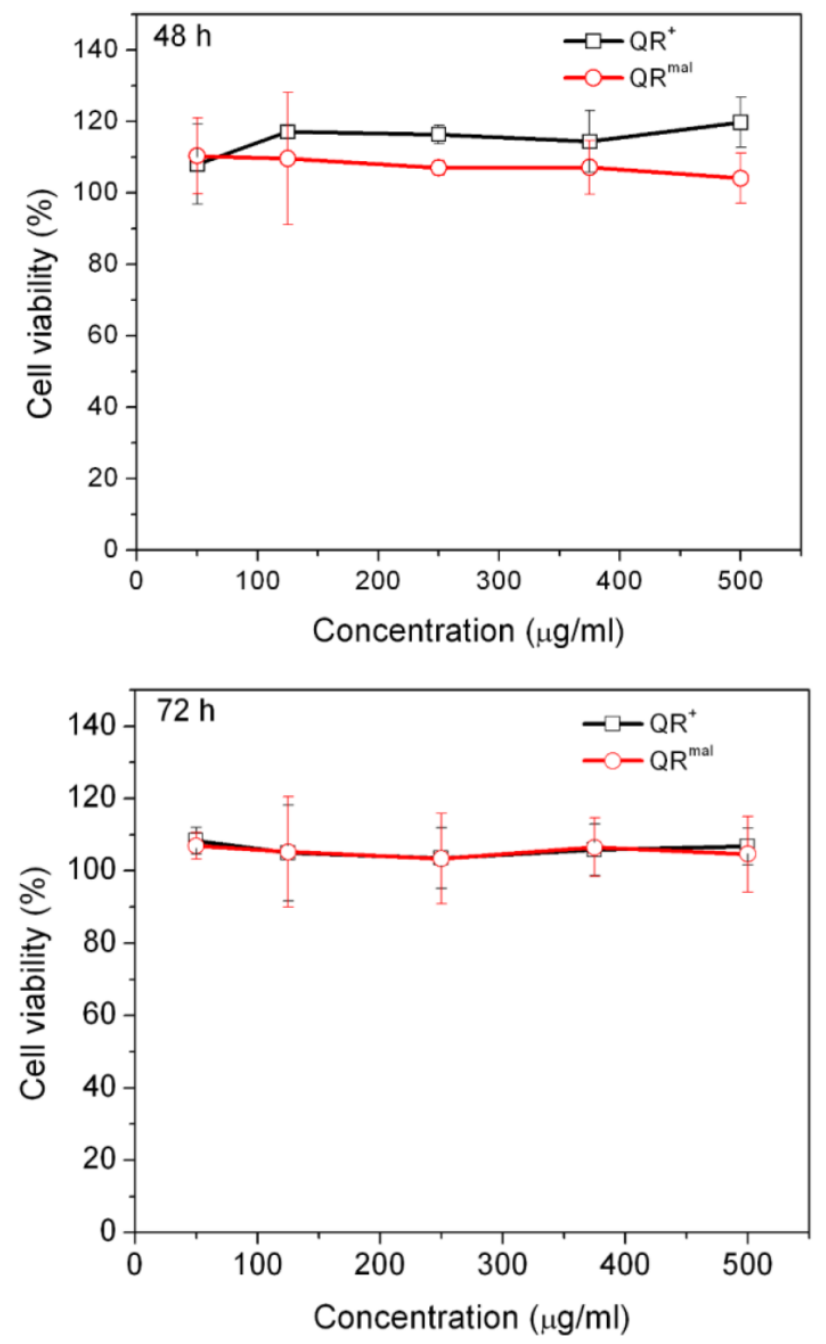

Figure 5. Cytotoxicity studies of positively charged $\left(Q R^{+}\right)$and maleimide modified $\left(Q R^{\text {mal }}\right) \mathrm{QR}$ micelles. DAN cells were treated with different concentration of micelles and incubated with (a) 48 hours and (b) 72 hours. 


\section{Conclusion}

We have demonstrated a phospholipid-based design of QR-siRNA nanoplex for gene therapy using different conjugation strategies. These multifunctional nanoparticles integrated imaging and gene delivery into single system which manifested low cytoxicity, high transfection efficiency and high biocompatibility. The suppression of DARPP-32 gene is not only implicated as siRNA delivery capabilities of QR nanomicelles but also an evidence for modulating the key components of dopaminergic signaling pathway. Although a high degree of challenge has been offered to the application of these nanomaterials for developing therapeutic agents for clinical use because of the Cd-based nature, our studies could provide useful guidelines for designing nanotechnology-based gene silencing carrier. One of the conceivable approaches would be replacing the Cd-based QR with heavy metal-free material such as silicon quantum dot. Our results suggest that a synthetic siRNA nanoplex harnesses the naturally occurring RNAi pathway in a manner that is consistent and predictable, thus making it an attractive nanotherapeutic that could be used not only for the treatment of drug addiction but also for a host of neurological disorders.

\section{Conflict of Interest}

The authors have declared that no conflict of interest exists

\section{Acknowledgement}

This study was supported by grants from the National Institute of Health [NIAID-RO1LM00972601; NIDA K01 DA024577; NIDA-1R21DA030108-01], the John R. Oishei and the Kaleida Health Foundations and in part by the Singapore Ministry of Education under Research Grant MOE2010-T2-2-010.

\section{References}

1. Walther C, Meyer K, Rennert R, Neundorf I. Quantum dot-carrier peptide conjugates suitable for imaging and delivery applications. Bioconjug Chem. 2008, 19: 2346-2356.

2. Prasad PN. Introduction to biophotonics. Wiley-Interscience: New York 2004.

3. Gao J, Chen K, Xie R, Xie J, Lee S, Cheng Z, et al. Ultrasmall near-infrared non-cadmium quantum dots for in vivo tumor imaging. Small. 2010, 6: 256-261.

4. Bhirde AA, Liu G, Jin A, Iglesias-Bartolome R, Sousa AA, Leapman RD, et al. Combining portable Raman probes with nanotubes for theranostic applications. Theranostics. 2011, 1: 310-321.

5. Law W-C, Yong K-T, Baev A, Prasad PN. Sensitivity improved surface plasmon resonance biosensor for cancer biomarker detection based on plasmonic enhancement. ACS Nano. 2011, 5: 4858-4864.

6. Yang M, Gao H, Zhou Y, Ma Y, Quan Q, Lang L, et al. 18F-Labeled GRPR agonists and antagonists: A comparative study in prostate cancer imaging. Theranostics. 2011; 1:220-229.

7. Lukianova-Hleb EY, Oginsky AO, Samaniego AP, Shenefelt DL, Wagner DS, Hafner JH, et al. Tunable plasmonic nanoprobes for theranostics of prostate cancer. Theranostics. 2011; 1:3-17.
8. Zhou Y, Li Y, Lu S, Ren H, Li Z, Zhang Y, et al. Gold nanoparticle probe-based immunoassay as a new tool for tetrodotoxin detection in puffer fish tissues. Sens Actuators B Chem. 2010, 146: 368-372.

9. Qian XM, Nie SM. Single-molecule and single-nanoparticle SERS: from fundamental mechanisms to biomedical applications. Chem Soc Rev. 2008, 37: 912-920.

10. Giljohann DA, Seferos DS, Prigodich AE, Patel PC, Mirkin CA. Gene regulation with polyvalent siRNA-nanoparticle conjugates. J Am Chem Soc. 2009, 131: 2072-2073.

11. Liu L, Law W-C, Yong K-T, Roy I, Ding H, Erogbogbo F, et al. Multimodal imaging probes based on Gd-DOTA conjugated quantum dot nanomicelles. Analyst. 2011, 136: 1881-1886.

12. Yong K-T, Roy I, Law W-C, Hu R. Synthesis of cRGD-peptide conjugated near-infrared CdTe/ZnSe core-shell quantum dots for in vivo cancer targeting and imaging. Chem Commun. 2010, 46: 7136-7138.

13. Law W-C, Yong K-T, Roy I, Ding H, Hu R, Zhao W, Prasad PN. Aqueous-phase synthesis of highly luminescent CdTe/ZnTe core/shell quantum dots optimized for targeted bioimaging. Small. 2009, 5: 1302-1310.

14. De La Zerda A, Zavaleta C, Keren S, Vaithilingam S, Bodapati S, Liu Z, et al. Carbon nanotubes as photoacoustic molecular imaging agents in living mice. Nat Nanotechnol. 2008, 3: 557-562.

15. Veiseh O, Kievit FM, Fang C, Mu N, Jana S, Leung MC, et al. Chlorotoxin bound magnetic nanovector tailored for cancer cell targeting, imaging, and siRNA delivery. Biomaterials. 2010, 31: 8032-8042.

16. Hom C, Lu J, Liong M, Luo H, Li Z, Zink JI, Tamanoi F. Mesoporous silica nanoparticles facilitate delivery of siRNA to shutdown signaling pathways in mammalian cells. Small. 2010, 6: 1185-1190.

17. Juliano RL, Ming $\mathrm{X}$, Nakagawa $\mathrm{O}, \mathrm{Xu} \mathrm{R}$, Yoo $\mathrm{H}$. Integrin targeted delivery of gene therapeutics. Theranostics. 2011; 1:211-219.

18. Pecot CV, Calin GA, Coleman RL, Lopez-Berestein G, Sood AK. RNA interference in the clinic: challenges and future directions. Nat Rev Cancer. 2011, 11: 59-67.

19. Whitehead KA, Langer R, Anderson DG. Knocking down barriers: advances in siRNA delivery. Nat Rev Drug Discov. 2009, 8: 129-138.

20. Davis ME, Zuckerman JE, Choi CHJ, Seligson D, Tolcher A, Alabi CA, et al. Evidence of RNAi in humans from systemically administered siRNA via targeted nanoparticles. Nature. 2010, 464: 1067-1070.

21. Chono S, Li S-D, Conwell CC, Huang L. An efficient and low immunostimulatory nanoparticle formulation for systemic siRNA delivery to the tumor. J Control Release. 2008, 131: 64-69.

22. Davis ME. The first targeted delivery of siRNA in humans via a self-assembling, cyclodextrin polymer-based nanoparticle: From concept to clinic. Mol Pharm. 2009, 6: 659-668.

23. Jean M, Smaoui F, Lavertu M, Methot S, Bouhdoud L, Buschmann MD, Merzouki A. Chitosan-plasmid nanoparticle formulations for IM and SC delivery of recombinant FGF-2 and PDGF-BB or generation of antibodies. Gene Ther. 2009, 16: 1097-1110.

24. Love KT, Mahon KP, Levins CG, Whitehead KA, Querbes W, Dorkin JR, , et al. Lipid-like materials for low-dose, in vivo gene silencing. Proc Natl Acad Sci U S A. 2010, 107: 1864-1869.

25. Jung J, Solanki A, Memoli KA, Kamei K-i, Kim H, Drahl MA, et al. Selective inhibition of human brain tumor cells through multifunctional quantum-dot-based siRNA Delivery. Angew Chem Int Ed Engl. 2010, 122: 107-111.

26. Elbakry A, Zaky A, Liebl R, Rachel R. Assembled gold nanoparticles for siRNA delivery. Nano Lett. 2009, 9: 2059-2064.

27. Qi L, Gao X. Quantum dot-amphipol nanocomplex for intracellular delivery and real-time imaging of siRNA. ACS Nano. 2008, 2: 1403-1410.

28. Yezhelyev MV, Qi L, O'Regan RM, Nie S, Gao X. Proton-sponge coated quantum dots for siRNA delivery and intracellular imaging. J Am Chem Soc. 2008, 130: 9006-9012.

29. Derfus AM, Chen AA, Min D-H, Ruoslahti E, Bhatia SN. Targeted quantum dot conjugates for siRNA delivery. Bioconjug Chem. 2007, 18: 1391-1396.

30. Jiang G, Park K, Kim J, Kim KS, Hahn SK. Target specific intracellular delivery of siRNA/PEI-HA complex by receptor mediated endocytosis. Mol Pharm.2009, 6: 727-737.

31. Lee H, Kim I-K, Park TG. Intracellular rrafficking and unpacking of siRNA/quantum dot-PEI complexes modified with and without cell penetrating peptide: Confocal and flow cytometric FRET analysis. Bioconjug Chem. 2010, 21: 289-295.

32. Song W-J, Du J-Z, Sun T-M, Zhang P-Z, Wang J. Gold nanoparticles capped with polyethyleneimine for enhanced siRNA delivery. Small. 2010, 6: 239-246.

33. Xia T, Kovochich M, Liong M, Meng H, Kabehie S, George S, et al. Polyethyleneimine coating enhances the cellular uptake of mesoporous 
silica nanoparticles and allows safe delivery of siRNA and DNA constructs. ACS Nano. 2009, 3: 3273-3286.

34. Fang $H$, Zhang K, Shen G, Wooley KL, Taylor J-SA. cationic shell-cross-linked knedel-like (cSCK) nanoparticles for highly efficient PNA delivery. Mol Pharm. 2009, 6: 615-626.

35. Wu J, Lizarzaburu ME, Kurth MJ, Liu L, Wege H, Zern MA, Nantz MH. Cationic lipid polymerization as a novel approach for constructing new DNA delivery agents. Bioconjug Chem. 2001, 12: 251-257.

36. Katas H, Cevher E, Alpar HO. Preparation of polyethyleneimine incorporated poly(d,l-lactide-co-glycolide) nanoparticles by spontaneous emulsion diffusion method for small interfering RNA delivery. Int J Pharm. 2009, 369: 144-154.

37. Bonoiu AC, Mahajan SD, Ding H, Roy I, Yong K-T, Kumar R, et al. Nanotechnology approach for drug addiction therapy: Gene silencing using delivery of gold nanorod-siRNA nanoplex in dopaminergic neurons. Proc Natl Acad Sci U S A. 2009, 106: 5546-5550.

38. Singh N, Agrawal A, Leung AKL, Sharp PA, Bhatia SN. Effect of nanoparticle conjugation on gene silencing by RNA interference. J Am Chem Soc. 2010, 132: 8241-8243.

39. Semple SC, Akinc A, Chen J, Sandhu AP, Mui BL, Cho CK, et al. Rational design of cationic lipids for siRNA delivery. Nat Biotechnol. 2010, 28 : 172-176.

40. Fu A, Gu W, Boussert B, Koski K, Gerion D, Manna L, et al. Semiconductor quantum rods as single molecule fluorescent biological labels. Nano Lett. 2006, 7: 179-182.

41. Kumar R, Ding H, Hu R, Yong K-T, Roy I, Bergey EJ, Prasad PN. In vitro and in vivo optical imaging using water-dispersible, noncytotoxic, luminescent, silica-coated quantum rods. Chem Mater. 2010, 22: 2261-2267.

42. Yong K-T, Qian J, Roy I, Lee HH, Bergey EJ, Tramposch KM, et al. Quantum rod bioconjugates as targeted probes for confocal and two-photon fluorescence imaging of cancer cells. Nano Lett. 2007, 7: 761-765.

43. Aaron JS, Greene AC, Kotula PG, Bachand GD, Timlin JA. Advanced optical imaging reveals the dependence of particle geometry on interactions between CdSe quantum dots and immune cells. Small. 2011, 7: 334-341.

44. Deka S, Quarta A, Lupo MG, Falqui A, Boninelli S, Giannini C, et al. $\mathrm{CdSe} / \mathrm{CdS} / \mathrm{ZnS}$ Double shell nanorods with high photoluminescence efficiency and their exploitation as biolabeling probes. J Am Chem Soc. 2009, 131: 2948-2958.

45. Sitt A, Salant A, Menagen G, Banin U. Highly emissive nano rod-in-rod heterostructures with strong linear polarization. Nano Lett. 2011, 11: 2054-2060.

46. Xu G, Yong K-T, Roy I, Mahajan SD, Ding H, Schwartz SA, Prasad PN. Bioconjugated quantum rods as targeted probes for efficient transmigration across an in vitro blood-brain barrier. Bioconjug Chem. 2008, 19: 1179-1185.

47. Yong K-T, Hu R, Roy I, Ding H, Vathy LA, Bergey EJ, et al. Tumor targeting and imaging in live animals with functionalized semiconductor quantum rods. ACS Appl Mater Interfaces. 2009, 1: 710-719.

48. Yong K-T, Roy I, Pudavar HE, Bergey EJ, Tramposch KM, Swihart MT, Prasad PN. Multiplex imaging of pancreatic cancer cells by using functionalized quantum rods. Adv Mater. 2008, 20: 1412-1417.

49. Cohen JL, Schubert S, Wich PR, Cui L, Cohen JA, Mynar JL, Fréchet JMJ. Acid-degradable cationic dextran particles for the delivery of siRNA therapeutics. Bioconjug Chem. 2011, 22: 1056-1065. 